\title{
Misunderstanding of ATP involvement in inflammation and stages of inflammation
}

\section{Explanation}

Inflammation is a disturbance of the normal metabolic events. The term 'inflammation' must not be mixed up with the term 'infection'. An infection is the hyponym of an inflammation following the aggression of bacteria, viruses, or fungus. ATP is considered a death signal starting inflammatory cascade in case of necrosis of cells. ${ }^{1,2}$ This view is propagated despite the publications showing ATP content into blood plasma compartment under normal, undisturbed physiological conditions. The sources of the ATP are the RBCs which constantly give away ATP out of their nucleotide pools. The resulting lack of adenosine is replaced while passing the liver sinusoids. Here the stocks of RBCs are refilled and ready to be transformed into ATP by glycolysis. ${ }^{3}$ Nevertheless, ATP is called a death signal. ${ }^{4}$ In this publication the author claims ' $\mathrm{P} 2 \mathrm{X}_{7}$ receptor requires a very high concentration $(>100 \mu \mathrm{M})$ of ATP for its activation and is thus a specific detector of large increases in [eATP], such as those that occur on cell death`. Necrotic cells are considered to expel ATP. ${ }^{1,5}$ The applied concentration exceeded the physiological amount of $1 \mu \mathrm{mol}$ $/ \mathrm{ml}$ full blood hundred-fold. Even in one of the latest publication 2012 of the New York Academy of Sciences researchers still believe that ATP is secreted by necrotic cells. ${ }^{6}$ Even though a publication by Welsch ${ }^{7}$ as early as 1994 in detail described the ATP content in the MCA and penumbra area after occlusion and reperfusion of the middle cerebral artery ${ }^{7}$ the apprehension is still propagated. From the pathophysiological point of view the release of ATP from necrotic tissue is not acceptable. These cells have undergone necrosis in the wake of total ATP depletion. The neuronal unit consists of endothelial cells, astrocytes, and neurons. In 1999 Magistretti published that neurons mainly metabolize lactate and are widely devoid of the glycolytic pathways. ${ }^{8}$ Whereas the astrocytes engage the endothelial cells by their processes and neurons the latter do not have access to the capillary system. ${ }^{9}$ Neurons do not metabolize glucose for energy production and therefore cannot produce ATP by glycolysis. Instead they are fed with lactate by astrocytes to meet metabolic demand and are extremely vulnerable to lack of oxygen. ${ }^{8}$

Welsch ${ }^{7}$ occluded the MCA (Arteria Cerebri Media) area in animal trials and ascertained the ATP content after $60 \mathrm{~min}$ and 24 hours of reperfusion. The necrotic area of the MCA contained minimal ATP, the residual ATP is derived from astrocytes on their way into apoptosis and not from the necrotic neurons. Therefore, the term 'necrosis' refers to a situation in which the ATP content is close to zero. In induced brain strokes in animals with differentiation of ATP content in the MCA area after occlusion and the penumbra show that the spot of first impact develops necrosis because of ATP depletion. ${ }^{7}$ Publications tell us that hypoxic cells release adenosine, not ATP, so how should necrotic cells gain or have in stock ATP? ${ }^{10}$ Welsch $^{7}$ reported that the penumbra zone develops apoptosis in a time frame of 24 to 48 hours, whereas the MCA zone undergoes the necrosis within minutes. Astrocytes in the penumbra zone dispose the glycolysis and a partial working ETC. In a state of apoptosis, the complex IV of the ETC maintains measure for the electric charge of the mitochondrial membrane. ${ }^{13}$ The reduced ATP production enables to maintain the mitochondrial membrane polarization and limited protein import

\author{
Volume 5 Issue 2 - 2018
}

Gerd Wasser
Henan University in Kaifeng, Facharzt für Innere Krankheiten,
Germany

Correspondence: Gerd Wasser, Henan University in Kaifeng, Facharzt für Innere Krankheiten, Franz-Marc-Str I2, 47506 Neukirchen-Vluyn, Germany, Tel +49 2845-9-68-66, Fax +492845-9-68-65, Email wasserdr@web.de

Received: June 07, 2018 | Published: July 02, 2018

during the mentioned 24 to 48 hours. ${ }^{11}$ How necrotic cells then should be capable to give away or expel ATP as death signal is not explicable. The main misunderstanding lies in the fact, that the role of ADP as the initiator of inflammation, of platelet aggregation, and even of smooth muscle activation is neglected ${ }^{12}$. The publications clearly explain that ATP is inhibiting the start of inflammation since the metabolic homeostasis is strictly ATP dependent. Even leucocyte adhesion and migration is not activated by ATP content, but ADP is involved in this major step towards cytokine production and migration in affected tissues. The misleading conclusions emerge out of the view that the full blood is on an $\mathrm{ATP}_{0}$ status instead of considering that living organism and the tissues never show a null energy level. ${ }^{12}$ The disturbance of the threshold levels of ATP signal disease and initiate inflammation. There is another reason for the misinterpretation regarding the ATP/ADP ratio. Since most researchers use the luciferase reaction to measure ATP the ADP amount is not considered. The exact luciferase reaction follows the rule: ATP+ADP light signal under luciferase to AMP and $\mathrm{PP}_{\mathrm{i}}$, without discriminating between ATP and ADP. This means that ATP is used and in the following kinase reaction the ADP to ATP reconstituted (information under: www.biaffin.comLumineszenzbasierte Assays). The proposed reaction reads as follows: xTP+ADP $=\mathrm{xDP}+\mathrm{ATP}$. But this will be the fact with the primarily existing ADP, too. If one compares the publications then one will find, that in most of the cases only the mutilated reaction ATP light to AMP and $\mathrm{PP}_{\mathrm{i}}$ is reported.

\section{Inflammation}

Inflammation is the course of metabolic, immunological, and repair events following injury or any other disturbance in the metabolic body balance. In general, the main stream medicine neglects the primary events leading to the first stage of inflammation due to lack of any medications which would abrogate the following stages. Since the inflammation pathways do not discriminate between different tissues we can predict the wake of pathophysiological events in brain, heart, or at any other body area. In other words, the conclusion is valid: if we can induce the same mechanism in the brain as we are able to observe in the skin we will have the same outcome. 


\section{Stage I of inflammation}

In case of an injury, in brain strokes or heart attacks, the spot of primary impact will undergo necrosis. In the heart with muscle cells capability to execute the glycolysis the area will follow the developing apoptosis. Inevitably necrosis will induce MOMP (Mitochondrial Outer Membrane Pore) opening. This event expels mitochondrial proteins, cytochrome $\mathrm{C}$, cardiolipin etc., located in the intermembrane gap, shedding the compounds into the cytoplasm. The lack of ATP opens ATP dependent potassium channels ( $\mathrm{K}_{\text {ATP }}$ channels). The opening of these channels shed the intercellular potassium into the interstitium and depolarizes the surrounding cell membranes. This event induces instant depolarization of the cells involved. The intracellular water content follows ions and is expelled b opening of aquaporins. The area is not completely cut off from blood supply in first time. The pressure of the edema later can cause ischemic areas in the tissue involved. But even supply of oxygen and glucose does induce any change since the cells are depolarized with the stunned metabolism. The only exception as mentioned before is mitochondria with maintained and limited ATP production. The other possibility to enhance ATP content via glycolysis is blocked by depolarization. There is only one possibility to reverse this stage of inflammation: to deliver ATP

\section{Stage 2 of inflammation}

The apoptotic pathway always is in line with the lack of ATP and the rise of ADP (Change of the ADP/ATP ratio) triggers leukocyte activation via purine receptors and the asymmetric distribution of ADP to monocytes, macrophages and leukocytes triggers migration to the affected area. ${ }^{13}$ The WBCs (white blood cells) will get attached by the production of ICAM1 to the endothelial layer rendering them ready for migration. ${ }^{14,15}$ The view that leucocytes sense ATP and direct their migration path by means of the ATP level in the environment is incorrect. ${ }^{1,16,17}$ This assumption does not consider that there is a constant level of ATP in the vasa signaling undisturbed metabolism. This reaction is in line with the sensing of energy depletion in cells. AMPK will be activated by ADP and not primarily by AMP in case of shrinking ATP pools..$^{18}$ Interleukins like TGF-b together with IL4, I16, and IL-15 and IL-23 promote inflammation via M1 macrophages and $T_{b} 17$ cells. Macrophages will collect and internalize the debris and clean the wound. Extracellular ATP inhibits the suppression of $\mathrm{T}_{\text {reg }}$ cells and abrogates inflammatory response. ${ }^{19}$ IL10 in concert with TGF-b blocks the proinflammatory response of $\mathrm{T}_{h} 1$ lymphocytes and the NFкB pathway. It is secreted by monocytes and $\mathrm{T}_{h} 2$-lymphocytes. In the intestine a lack of IL10 is responsible for inflammatory intestinal diseases. ATP induces permeabilization of macrophages after $40 \mathrm{msec}$ of ATP application. This phenomenon may be inducing the forming of giant cells by opening pores in the size of gap junctions ${ }^{20}$ which in turn leads to engulf debris.

\section{Stage 3 of inflammation}

Resident tissue fibroblasts and myofibroblast derived from pericytes in a specific conformation will produce fibrocytes which build the lasting scar in secreting elastic and collagenous fibrils. In neuronal tissue myofibroblasts derived from pericytes will close the defect. ${ }^{21}$ With this involvement of fibroblast and myofibroblasts, respectively, the inflammation comes to a hold by production of the lasting scar.

\section{Conclusion}

The lack of ATP and the risen ADP levels determine the course of inflammation and not the seemingly soared ATP levels in the interstitium. It is widely accepted that ATP serves as a danger molecule activating the neighboring cells to start the inflammatory cascade. As early as 1990 the Annals of the New York Academy of Sciences described the basic knowledge about the action of extracellular delivered ATP. ${ }^{22}$ Later publications reported the nucleotide content in ischemic areas like artificial induced brain stroke in animal trials. ${ }^{7,23}$ Since then not the ATP amount but the ATP to ADP ratio signals stable metabolic conditions or in case of a shift to higher ADP amounts starts the inflammatory cascade. Since researchers still use the luciferase reaction to quantify nucleotide content misinterpreting it as ATP content the ATP/ADP ratio is neglected. The effect on still ongoing research is that laboratories use 100-to 500-fold dosage of artificially administered ATP to have a closer look the reaction of receptors or on transformation of macrophages into dendritic cells and to draw conclusions on physiological or patho-physiological events. ${ }^{4,24}$

\section{Acknowledgements}

None.

\section{Conflict of interest}

The author declares there is no conflict of interest.

\section{References}

1. Linden Joel. Cell biology purinergic chemotaxis. Science. 2006;314(5806):1689-1690

2. McDonals Braedon, Pittman K, Menezes GB, et al. Intravascular danger signals guide neutrophils to sites of sterile inflammation. Science. 2010;330(6002):362-366.

3. Rapaport E. Mechanisms of anticancer activities of adenine nucleotides in tumor-bearing hosts. Ann N Y Acad Sci. 1990;603:142-150.

4. Trautmann A. Extracellular ATP in the immune system: more than just a danger signal. Sci Signal. 2009;2(56):pe6.

5. Szabo Csaba, Pal Pacher. The outsiders: emerging roles of ectonucleotidases in inflammation. Sci Transl Med. 2012:4(146):146ps14.

6. Heiss. Wolf-Dieter: The ischemic penumbra: how does tissue injury evolve? Ann N Y Acad Sci. 1268:26-34.

7. Welsch Frank A. Regional expression of immediate-early genes and heatshock genes after cerebral ischemia. Annals of the New York Academy of Sciences.1994;723:318-327.

8. Magistretti, Luc Pellerin, Douglas L Rothman,et al. Energy on Demand Science. 1999;283(5401):496-497.

9. Del Zoppo G. Stroke and neurovascular protection. $N$ Engl J Med. 2006;354(6):553-555

10. Vollmar B, Menger MD. The hepatic microcirculation: mechanistic contributions and therapeutic targets in liver injury and repair. Physiol Rev. 2009;89(4):1269-1339.

11. Stappenbeck TS, Miyoshi H. The role of stromal stem cells in tissue regeneration and wound repair. Science. 2009;324(5935):1666-1669.

12. Slakey Linda L, Ellen L Gordon, Jeremy D Pearson. A comparism of ectonucleotidases activities on vascular endothelial and smooth muscle cells. Annals of the New York Academy of Sciences. 1990;603:365-379. 
13. Chen Y, Corriden R, Inoue Y, et al. ATP release guides neutrophil chemotaxis via p2y2 and a3 receptors. Science. 2006; 314(5806):17921795 .

14. Alvarez Jorge Ivan, Dodelet-Devillers A, Kebir H, et al. The hedgehog pathway promotes blood-brain barrier integrity and cns immune quiescence. Science. 2011;334(6063):1727-1731.

15. Engelhardt Britta. Blood-brain barrier differentiation. Science. 2011;334(6063):1652-1653.

16. Yu Chen, Corriden R, Inoue Y, et al. ATP release guides neutrophil chemotaxis via p2y2 and a3 receptors. Science. 2006;314(5806):17921795 .

17. Vinatha Sreeramkumar, Adrover JM, Ballesteros I, et al. Neutrophils scan for activated platelets to initiate inflammation. Science. 2014:346(6214):1234-1238.

18. Czupryn A, Zhou YD, Chen X, et al. Transplanted hypothalamic neurons restore leptin signaling and ameliorate obesity in $\mathrm{db} / \mathrm{db}$ mice. Science. 2011;1133-1137.
19. Stam Jan MD. Thrombosis of the cerebral veins and sinuses. $N$ Engl J Med. 2005;352(17):1791-1798.

20. Steinberg Thomas H, Buisman HP, Greenberg S, et al. Effects of Extracellular ATP on Mononuclear Phagocytes. Annals of the New York Academy of Sciences. 1990;603:1221-129.

21. Göntz Christian, Dias DO, Tomilin N, et al A pericyte origin of spinal cord scar tissue. Science. 2011;333(6039):238-242.

22. Biological Actions of Extracellular ATP. Annals of the New York Academy of Sciences. 603:1-542.

23. Cellular, Biochemical, and Molecular Aspects of Reperfusion Injury. Ann N Y Acad Sci. 723:1-502.

24. Marine Bretou, Sáez PJ, Sanséau D, et al. Lysosome signaling controls the migration of dendritic cells. Sci Immunol. 2017:2(16). 